\title{
Introduction: Connecting Digital Humanities and Medical History through Viral Networks
}

\author{
E. THOMAS EWING AND KATHERINE RANDALL
}

Milestones in the development of a networked understanding of disease transmission are also milestones in the history of medicine. figure 1, adapted from a 1984 article in the American Journal of Medicine, demonstrates how the earliest research on the emerging AIDS epidemic used network analysis to identify relationships among patients who were spreading this disease. ${ }^{1}$ This article relied on interviews with nineteen patients about their sexual partners, which generated the forty circles connected by lines indicating sexual exposure. One patient, marked as Patient 0 , located at the center, was connected directly to eight patients and, through a second link, to another eight. Based on network analysis of clusters of infected patients, the article, written by a team of leading experts in the study of this new and frightening disease, endorsed the recommendation issued less than a year earlier by the Centers for Disease Control and Prevention: "Members of high-risk groups should be aware that multiple sexual partners increase the probability of getting AIDS."

At the time, and even more so in subsequent years, this single network visualization functioned on multiple levels: instrumental as a tool for epidemiology, limited as an analytical operation, powerful in its cultural impact, and tragic in its human costs. This chart 


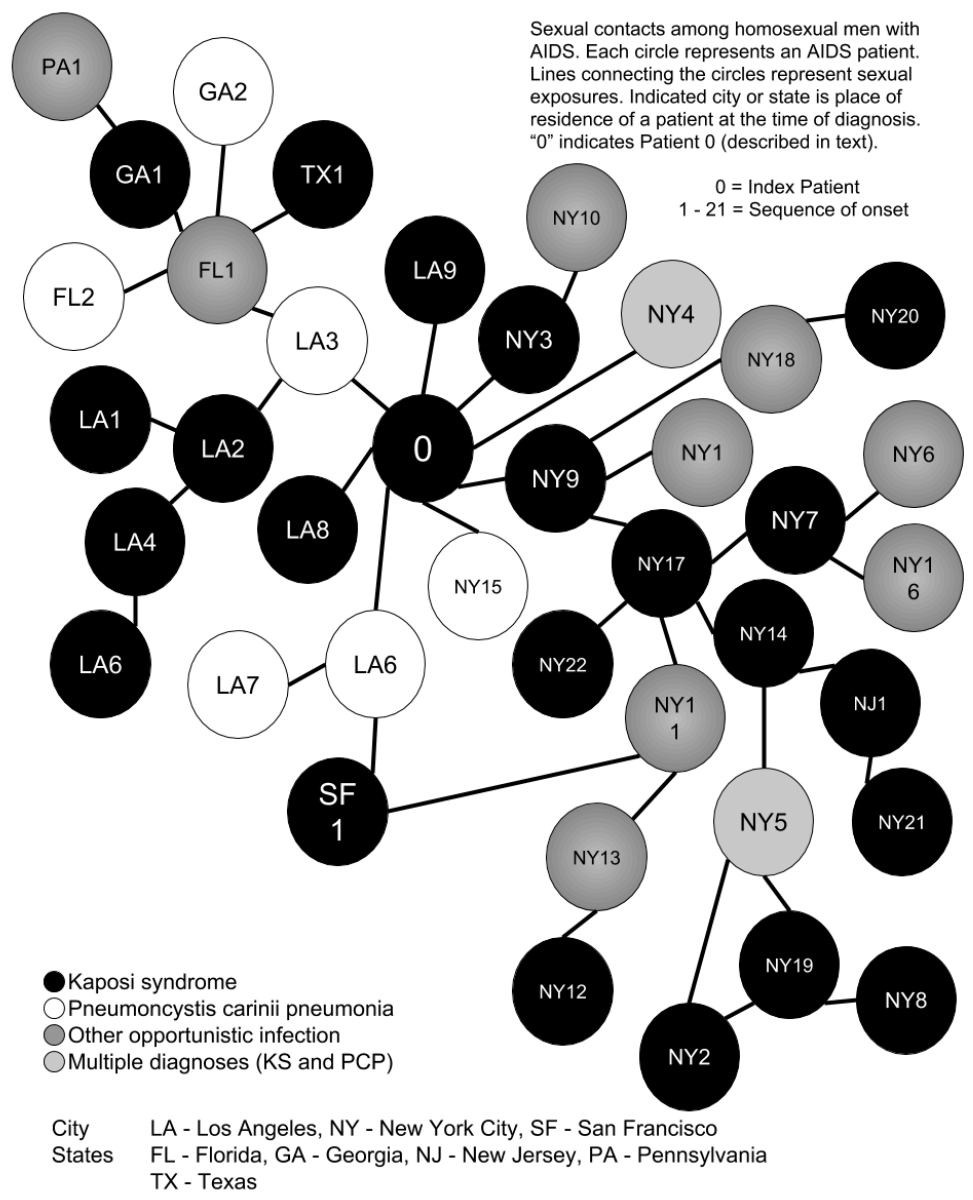

Figure 1: Network Analysis of AIDS Patients

resulted from a relatively small data sample, interviews with less than twenty individuals (or with close friends and family members, in the case of deceased subjects), in a year in which AIDS is estimated to have killed approximately four thousand people in the United States. The conclusions were expressed in guarded, clinical language, yet in practice may have reinforced hostility towards those engaged in what was then called risky behavior. Most 
significantly, the man connected to all the other patients was originally called Patient $\mathrm{O}$ (the letter $\mathrm{O}$ was an abbreviation for Out of California), but this individual was identified as Patient 0 (in this diagram), and then popularized-and vilified-as Patient Zero, the alleged starting point for the spread of HIV/AIDS in the United States. ${ }^{2}$ In other words, a network map used as an analytical tool to represent disease transmission between individuals was transformed into a symbol of a certain kind of behavior that fit into dominant narratives of the era in ways that continue to shape perceptions of disease in popular, scholarly, and even scientific contexts. The human beings whose behaviors were reduced to circles and lines, including Gaetan Dugas, the man later identified as Patient Zero, mattered as individuals, but also as nodes of a network connected not only to each other but to millions of AIDS victims around the world in the decades that have followed since this network was identified in the early 1980s.

This illustration serves as an effective way to introduce the subjects, partnerships, collaborations, and processes that produced the chapters in this volume. All of these chapters deal with topics, themes, and problems in medical history, yet their chronological, geographical, and thematic perspectives range widely and vary considerably. Just as the metaphor of the network illustrates connections while recognizing distinctiveness, these chapters share a common approach informed by network analysis; yet the types of data, the tools used, and the outcomes observed also varied considerably. Most important, whereas the AIDS network diagram simplified complex human relationships in ways that permitted and even encouraged distortions premised on stereotypes, each chapter in this volume engages critically, thoughtfully, and productively with the value of network analysis as an analytical tool. In other words, even as the AIDS network diagram inspired critical thinking about connectivity, it was consistently and creatively challenged, revised, and ultimately re-imagined as a way to think about both networks in medical history and networks among scholars. 
The Viral Networks project thus approaches networks as an object of study, a tool for analysis, a framework for collaboration, and a means of scholarly communication. The scholars who participated in this project examined networks in medical history even as they became "nodes" in a network of scholars engaged in collaborative learning. The workshop, inspired by models of networked pedagogy, brought these scholars into a connected series of activities that began with reading proposals, included one face-to-face and two virtual conferences, and ended with final edits on revised chapters. This collaboration helped address many of the issues that came up for each author as they wrote for a wider audience, including questions about how much historical content to include or cut in order to focus the paper on methodology. In essence, the authors in this collection spent months not only on their own papers but on guiding and critiquing the papers of their co-collaborators. The chapters should therefore be understood and read as a fully networked project, not as chapters written individually and placed together.

The tools of network analysis made possible by the digital humanities were enhanced by more traditional humanities methods of close reading, contextual analysis, and layered interpretation. Each chapter author was a node in this network, connected to the other authors by the experience of reading, editing, and evaluating each other's work, yet also connected by the shared experience of using networks as a tool for historical analysis. Finally, each author studied the operation of networks in medical history as a relationship among ideas, people, institutions, or language. Much as the first visualization of relationships among AIDS patients represented a reality of social interactions even as it became a tool for understanding this disease, the Viral Networks workshop created a relationship among scholars working collaboratively toward a shared outcome of understanding the place and significance of networks in medical history by integrating approaches from the digital humanities and network analysis. 
The Viral Networks project marks the convergence of three important trajectories: first, the fact that networks are an essential aspect of living the human experience; second, the development of more accessible and powerful network analysis tools; and third, the opportunity to make scholarship more collaborative and accessible through digital humanities tools. As illustrated in these chapters, networks were an essential aspect of the human experience in the form of communication between and among individuals, the operation of medical teams, the debate over the meaning of concepts, the use of tools for diagnosis and treatment, and personal appeals based on shared narratives of experience and established frameworks of order. Networks were central to the human experience; studying networks is thus an essential tool and step in the process of understanding the human experience. As humanities scholars, the participants in this workshop collectively and individually examined networks as an aspect of the experience of the people and processes central to human experiences. Some scholars were committed to network analysis from the inception of their studies; others used the opportunity to participate in this workshop as an inspiration to explore their subjects in a new way.

A recurring question during the Viral Networks project has been, "What can a network show you that another type of analysis can't?" The chapters in this volume demonstrate what a network analysis can reveal, but also how a network analysis can help a humanities scholar approach a problem in a different way, or understand what is missing in their sources or interpretations. A network methodology may not be the most appropriate to answer every research question and every project. But any humanities scholar can use network analysis when it is appropriate, and our intent with this collection was to demonstrate what that might look like. The scholars who contributed to this collection are all studying topics in the history of medicine-the common denominator for the Viral Networks project-but they vary in research area, familiarity with network processes, and level of comfort with network analysis software. As one of our outside readers for this collection 
commented, each chapter "represents work in progress, opening a window onto the author's work at a particular moment in its development." The chapters are snapshots of a research process, meant in many cases to demonstrate methodology-in-process as scholars deliberately work through what network tools and techniques mean for their project, what they learned from their use, and how their work has changed because they have self-consciously applied this approach.

In chapters one through three, the authors navigate the new terrain of network methodology as traditional historians, documenting research journeys that are valuable to other humanist scholars who are unfamiliar with network methods and tools. In chapter one, Runcie brings academic conversations regarding postcolonialism and the ethics of using colonial records in constructing historical narratives to network analysis. Networking healthcare teams in colonial Cameroon, Runcie demonstrates how varying data inputs in data visualizations can re-center the focus on Cameroonian medical auxiliaries and away from French colonist medical authorities. Smith's chapter two essay demonstrates how networks of psychiatrists, hospitals, and the government worked to maintain segregation in 1960s Alabama, while also tracing the process (and difficulty) of moving from analog to digital history work. Smith shows how historians can build upon hand-drawn mapping of people, places, and events to using digital tools with a more specific focus. In chapter three, Sorrels explores the intersections between allopathic and alternative medicine by networking citation data between practitioners, asking what can be learned about how these two seemingly disparate sects interact from where and how frequently their practitioners published. Sorrels also challenges new digital humanists to navigate the line between reducing the complexity of humanistic research and producing the specific questions and bounded data required for network analysis.

A concern raised in these first three chapters is how to determine what data should be included in the analysis. The authors of 
chapters four and five address this concern in more depth, walking readers through the process of preparing archival materials for network analysis. Engelmann develops in chapter four a genre of early epidemiology outbreak reports, arguing that pinpointing the concepts involved in data extraction for network analysis is in itself an epistemological exercise that opens up new ways of seeing for the historian. Though Engelmann does not use this data to create a network visualization in this paper, he theorizes multiple ways in which the data could be used in a revelatory network analysis. In chapter five, DiMeo and Ruis walk readers through an example of how to take a digitized data set-in this case, the mid-seventeenth century Hartlib papers-and determine how to ask the right research questions in order to glean the appropriate data to then feed into the epistemic network analysis. They challenge researchers to think about what makes network analysis appropriate for a project, how to determine which elements of the data should be included or excluded, and how a historical data set must be understood for a mixed-methods approach, among other considerations. They deliberately focus on the "work in progress" stage of a network analysis project in hopes of demystifying the process for historians new to digital methods.

In chapters six through nine, the authors offer reflections based on the results of their networks. Cottle's chapter six looks at the epistolary networks that emerge in the early-twentieth-century correspondence between two academic women, focusing both on what Cottle terms "macroscopic" and "microscopic" anatomy. While macroscopic anatomy is the level of analysis that comes from traditional historical research, Cottle argues that digital visualizations of connections and themes (microscopic anatomy) can help historians trace connections and networks among people, places, and ideas in written correspondence. While other contributors focus on specifying a research question for a network analysis project, in chapter seven Archambeau demonstrates how the unexpected results in a network analysis can change the trajectory of a research question and challenge assumptions a 
researcher may have about data. Archambeau uses plague references made in witness testimonies during a canonization inquest in fourteenth-century Provence to look for characteristics and patterns in how people remember and engage with plague events. In chapter eight, Ruis maps the shifting conception of nutrition over the nineteenth and twentieth centuries, demonstrating how the computer modeling of epistemic network analysis can be used by historians as a tool of macrohistorical analysis to complement traditional close reading. Ruis argues that using this kind of mixed-methods approach can be a way to expand historical understandings of-and create new arguments about-the past. Finally, in chapter nine, Phillips uses network analysis as an exploratory tool, demonstrating through his study of how a core group of researchers at the National Institutes of Health brought statistics into medicine in the mid-twentieth century that historical researchers should not be afraid of thinking in networked terms, though there is no one precise way to apply network tools to archival research.

While the approaches to network methodology used by the authors in this volume vary widely, what is reassuring to network newcomers is that none of them is wrong. Network analysis, like the networks themselves, is often more flexible and open-ended than we might think. This flexibility in network methodology is both encouraging, in that it has room to accommodate humanist scholars, and daunting, in that it can take many shapes for different ends. As many of the authors demonstrate, using network methodology requires critical perspective and judgment in determining what data to include or exclude, and in finding the appropriate way to contextualize what the network shows (or doesn't show). Fortunately, humanist scholars are well-suited to these tasks, being intimately concerned with issues of how ideas spread, how people are connected, and who read/says what to/ by whom. The keynote speaker at the workshop, Teresa MacPhail, illustrated this approach to network analysis by connecting historical examples of epidemics to present and future strategies 
by government agencies and non-governmental organizations for dealing with epidemic disease. Using her analytical methods as an anthropologist, MacPhail focused on the human beings within these medical establishments who gather information, evaluate evidence, make recommendations, and deal with the consequences. By focusing on the human element of networks, MacPhail's approach set the tone for the chapters to emulate this interdisciplinary perspective on digital humanities and medical history.

For the methodology-with which many of the Viral Network participants were previously unfamiliar-we benefited greatly from the assistance of data visualization and network scholars who were critical in demonstrating that networks have great potential as well as significant limitations as a tool for digital humanities projects. At the workshop's opening session, Amy Nelson of the Virginia Tech Department of History described how networked learning can enhance both the collaborative and individual contributions of students to research projects. The networked nature of learning is closely connected to the goals of public learning and open access, which provides further reinforcement to this project's emphasis on both the openness of the research process and the accessibility of the research outcomes. Ryan Cordell of the Department of English at Northeastern University described the Viral Texts project and how it explores networks of information constructed by American newspapers in the nineteenth century. By focusing on the changing nature of authorship in the interstices of these networks, this presentation provided a model for this workshop's emphasis on collective reviewing and editing of texts. Finally, Samarth Swarup of the Biocomplexity Institute at Virginia Tech discussed tools for network analysis used by computational analysts across fields, including epidemiology, for understanding and predicting large scale patterns of change. A common theme in all three presentations was the importance of recognizing the humans at the center of the networks, a theme that also connects all the chapters in this book. Finally, Nathaniel Porter, the Social Sciences Data Consultant at University Libraries at Virginia Tech, provided 
guidance to the individual scholars, worked with colleagues to develop data visualizations in this volume, and contributed a chapter that discusses the advantages of integrating network analysis with humanities scholarship. Throughout the two days of the workshop, these scholars, as well as observers from the National Institutes of Health National Library of Medicine and National Center for Biomedical Information, contributed their critical perspectives on the chapters and made recommendations for expanding, refining, or reconfiguring tools in order to better understand source materials and analytical questions.

As the volume editors, we can step back from the workshop and subsequent discussions of chapters to identify key themes that illustrate the scholarly contribution of this volume as a whole: there are connections that may not mean causation; the research questions in a network approach should be finely targeted; not all the complexities of the data can be shown in a single network; and there is bias in a network due to what is preserved, coded, and collected. In some cases, the authors and consulting scholars were able to find strategies to address and overcome these challenges. In other cases, the authors used these concerns to engage critically with the limits of using networks as an analytical tool. The Viral Networks workshop and the contributions to this volume demonstrate how digital network methodology expertise and humanities scholarship can work together to advance and provide new insights that benefit both fields.

"We experience life as a narrative, not as a map and certainly not as a network," was the deliberately provocative claim made in 2016 by Mushon Zer-Aviv, in the equally provocatively entitled post, "If everything is a network, nothing is a network." ${ }^{3}$ As co-editors of this volume, we also experienced this process as a narrative: the call for papers that allowed authors to propose topics; a first virtual meeting to review abstracts; two days of intensive discussion at the National Library of Medicine with contributing authors, consulting scholars, and observers; the substantial revision of chapters, which were then reviewed by other contributing authors; another virtual 
conversation to discuss recommended edits; and the final stages of editing, proofing, and publishing this volume. In contrast to ZerAviv's claim, however, we also experienced this process as a network: the intellectual connections with scholars, the conversations in the conference room of the National Library of Medicine, and the shared editing space of folders, documents, and virtual discussions. Narratives and networks are not mutually contradictory; networks can be experienced as narratives and narratives can be experienced as networks.

Defining and mapping networks is central to several influential digital humanities projects, including Viral Texts: Mapping Networks of Reprinting in 19th Century Newspapers and Magazines, Colored Conventions: Bringing Nineteenth Century Black Organizing to Digital Life, Six Degrees of Francis Bacon, and Mapping the Republic of Letters. ${ }^{4}$ All of these projects illustrate how network analysis, using easily accessible tools and digitally curated data, can become an insightful and accessible tool for humanities scholars. Network analysis is popular in digital humanities projects because scholars in fields such as literature, history, and anthropology have recognized connections among individuals to be powerful forces in shaping experiences, values, and relationships; yet these networks can also be transformed into data in ways that can be analyzed by computer scientists and others in data fields. The proliferation of visualizations in these projects illustrates the potential of network analysis to transform the textual evidence valued by humanities scholars into the charts, diagrams, and webs more familiar to scholars in computational fields. These projects directly address key questions for the humanities using new tools that provide fresh perspectives on available evidence: How do ideas spread among people and across communities? How can the diversity of participants be recognized while also exploring the commonality of ideas? How did networks allow ideas to be simultaneously debated at the more sophisticated levels while also penetrating every level of society in the form of published texts and spoken words? Yet the illustration of these connections has not always sufficiently engaged 
with the core humanities challenge of understanding and interpreting meaning; or, to use language from the computational fields, the correlations among people, ideas, and places has not always been accompanied by sufficient attention to causation. The presence of network analysis in the digital humanities has been intellectually powerful in ways that have generated significant projects and inspired new research fields, yet the challenge is to move beyond these specific case studies to understand the value of network analysis as a research tool connecting disparate fields.

Viral Networks builds on these remarkable examples of successful implementation of network analysis in the digital humanities, but its larger goal has been to cultivate and support a broad community of contributing scholars, drawn from a range of institutions, thus building a model of collaborative and networked research and writing that can inspire more projects in the future. We encourage readers of this volume to take advantage of the flexibility of digital scholarly publication. The chapters, indeed the entire volume, can be read in a linear fashion, starting with the introduction and proceeding through each chapter, in either the digital form or a print edition. Yet readers may also choose to read across layers, moving from the text of the chapters to the networked diagrams to the data for each chapter, thus finding that the act of reading follows a networked structure similar to that experienced by workshop participants. These chapters should also be read as works-in-progress; in effect, as part of a networked conversation among the individual chapter authors, the workshop participants, and the readers of this volume. In this sense, the chapters are not a final definitive word, but rather an effort to engage both medical historians and digital humanities in continuing to think creatively and critically about the interpretive value of network analysis as a tool, a process, and a metaphor.

The cover image for this volume, a photograph of a training school for nurses in Illinois (figure 2), provides evidence that networking in medical history is neither a new phenomenon nor a product of visualization tools. ${ }^{5}$ Professional associations of nurses and 
physicians, conferences, and training programs have emerged over the centuries as ways to connect medical personnel, patients, and the general public. ${ }^{6}$ The more formal gathering of nurses illustrated

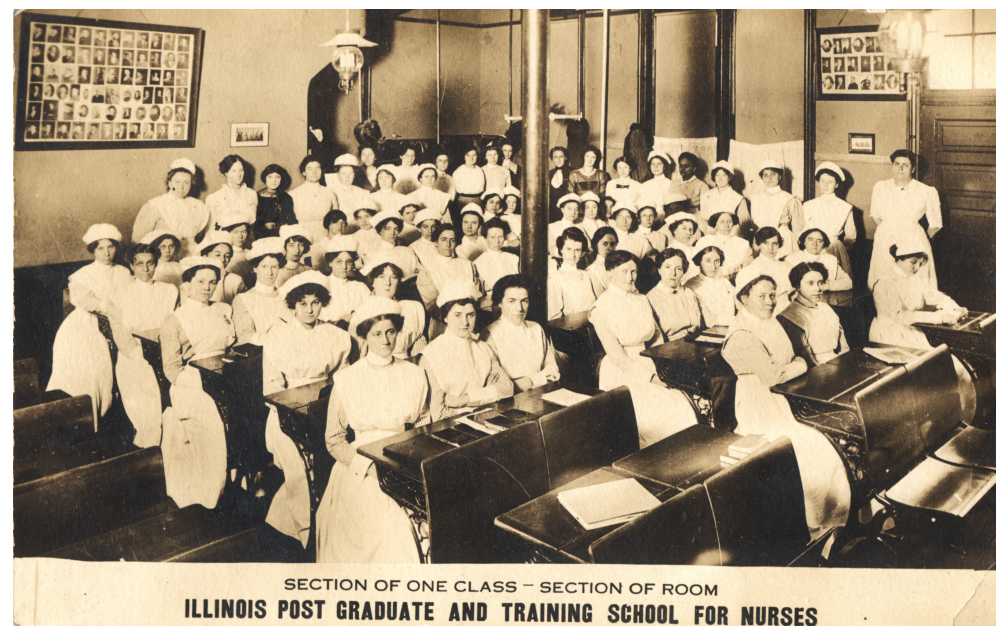

Figure 2: Illinois Post Graduate and Training School for Nurses

in this photograph became increasingly widespread in nineteenth and twentieth centuries, and serve in some ways as a model for the Viral Networks workshop hosted by the National Library of Medicine, funded by the National Endowment for the Humanities, and organized by Virginia Tech. Like the AIDS diagram in figure 1 , this photograph captures a moment in time, with no indication of the specific steps that brought these individuals together, and certainly no way of predicting whether the connections made in this training program lasted in the months, years, and even decades ahead-or whether they ended as soon as the training school came to an end. Yet this photograph reminds scholars that even in a digital age tremendous value remains in the capacity to bring participants together in a single room, to discuss common research interests, to learn from experts and from each other, and to leave the session better educated and more committed to professional activities. We hope this collection is useful to medical historians 
looking for new tools to understand research topics, to humanities scholars looking for ways to acquire and apply new analytical tools, or to students at any stage of learning who are interested in how networks might add new dimensions to their research.

\section{Endnotes}

1. D. M. Auerbach, et al, "Cluster of Cases of the Acquired Immune Deficiency Syndrome: Patients Linked by Sexual Contact," American Journal of Medicine, 76, (1984): 488.

2. The naming of Patient Zero reached the broadest public audience in Randy Shilts, And the Band Played On. Politics, People, and the AIDS Epidemic (New York: St. Martin's Press, 1987). For historical analysis of this mis-identification, see Richard A. McKay, "Patient Zero': The Absence of a Patient's view of the Early North American AIDS Epidemic," Bulletin of the History of Medicine 88 (2014): 161-194; idem, Patient Zero and the Making of the AIDS Epidemic (Chicago: University of Chicago Press, 2017); Jon Cohen, "'Patient Zero' No More," Science, March 4, 2016.

3. Mushon Zer-Aviv, "If everything is a network, nothing is a network," https://visualisingadvocacy.org/blog/if-everything-network-nothing-network, accessed May 2018.

4. The Viral Texts Project: Mapping Networks of Reprinting in 19th Century Newspapers and Magazines, http://viraltexts.org/; Colored Conventions: Bringing Nineteenth Century Black Organizing to Digital Life, http://coloredconventions.org/; Six Degrees of Francis Bacon, http://www.sixdegreesoffrancisbacon.com/; Mapping the Republic of Letters, http://republicofletters.stanford.edu/.

5. Illinois Post Graduate and Training School for Nurses: section of one class section of room (date of collection: 1914). Courtesy of the U.S. National Library of Medicine. https://collections.nlm.nih.gov/catalog/nlm:nlmuid-101611030-img.

6. "American Nursing: An Introduction to the Past," University of Pennsylvania School of Nursing: https://www.nursing.upenn.edu/nhhc/ american-nursing-an-introduction-to-the-past/; "Nursing History in Illinois," University Library, University of Illinois, Urbana-Champaign: https://researchguides.uic.edu/nursinghistory. 